\title{
On STEM of tectonic stress fields in tsunami regions
}

\author{
J. Irsa ${ }^{1,2}$ \& A. N. Galybin ${ }^{1,3}$ \\ ${ }^{1}$ Wessex Institute of Technology, Southampton, UK \\ ${ }^{2}$ Florida Institute of Technology, USA \\ ${ }^{3}$ Institute of Physics of the Earth, RAS, Moscow, Russia
}

\begin{abstract}
We apply the STEM (Stress Trajectories Element Method) to investigate changes in the stress states as a result of a great earthquake. Two regions are considered: 1) the region affected by the great Sumatran earthquake $\left(26^{\text {th }}\right.$ of December 2004, 9.1M), taking into account data collected before and after the event separately; 2) the region where the recent Japanese earthquake $\left(11^{\text {th }}\right.$ of March 2011, 9.0M) occurred. The latter is analysed taking into account data collected before the event only while the former case allows one to consider the changes in data due to the significant increase of measurements since the 2004 event. The analysis is based on the stress orientation data from the World Stress Map (WSM) database and the geometrical model of tectonic plate boundaries. The method has proved to be able to recover the stress fields from this type of data with satisfactory accuracy. The modelling utilizes the Trefftz type approach for the complex potentials in plane elastic problems and assumes continuity of the stress vector across the tectonic plate boundaries. As a result of the modelling we obtain stress trajectories, maximum shear stress and mean stress, and the plate driving forces expressed in terms of normal and tangential stresses. The results for the two time subsets revealed changes in the stress patterns induced by the 26/12/2004 event and moreover the stress pattern before the great Sumatran Earthquake was very similar to the one near Japan.
\end{abstract}

Keywords: world stress map, stress reconstruction, stress changes, Sumatran earthquake, Japan earthquake. 


\section{Introduction}

The great Sumatra-Andaman earthquake on the 26.12.2004 with the magnitude of 9.1 is the $3^{\text {rd }}$ greatest earthquake since 1900 , and the Japanese earthquake on the 11.03.2011 is the $4^{\text {th }}$ largest [4]. The motivation of this study is to investigate the changes in tectonic stress fields induced by the 26.12.2004 event and also analyse the region where the Japan earthquake occurred, based on the developed numerical method, STEM [3]. The first area for the analysis is the Sunda trench region where data before and after the event are available. The second is the Japan region where data are available only before the date of the event.

The approach uses the data on stress orientations from the World Stress Map database [1] and the tectonic plate boundaries database PB2002 [2]. The numerical algorithm is based on the previous study [3] but it has been significantly modified in order to take the multiple plate margins into account. Using the modified STEM approach we determine not only the stress state inside the tectonic plates but also the boundary forces on the tectonic plates. It is assumed that the tangential and normal stresses are continuous across the plate margins.

The method is applied to model the stress states of the following regions: Sunda region (longitude $80 \mathrm{E}-110 \mathrm{E}$, latitude $20 \mathrm{~S}-10 \mathrm{~N}$ ) and Japan region (longitude $125^{\circ} \mathrm{E}-150^{\circ} \mathrm{E}$, latitude $27^{\circ} \mathrm{N}-45^{\circ} \mathrm{N}$ ). The results allow us to observe the stress changes after the great Sumatran earthquake and also to compare the two regions based on their stress patterns.

\section{Methodology}

\subsection{Stress orientations data and plate boundaries data}

The stress orientations data are taken from the WSM database, release 2008, containing over 21000 data. Most of the data are of quality A-C, which is considered to have accuracy $\pm 25^{\circ}$ in the stress orientation [1]. A single entry in the database shows location, azimuth of the major principal stress, quality, depth, stress regime, date/time, method, magnitude of the earthquake (if from earthquake), some other information as focal mechanism, etc. The Sumatra region includes of 854 data, which is further divided into two groups of 269 data for the CASE 1 (before event) and 585 data for the CASE 2 (after event). The Japan region includes 982 data (all before the event).

The digital boundaries of the tectonic plates are taken from the database PB2002 [2], describing 52 plates. The Bird's data and WSM data for the Sumatra region are shown in Figure 1, and for the Japan region in Figure 2.

\subsection{Assumptions in modelling}

The following assumptions are accepted:

The lithosphere is considered to be elastic and of constant (effective) thickness. 


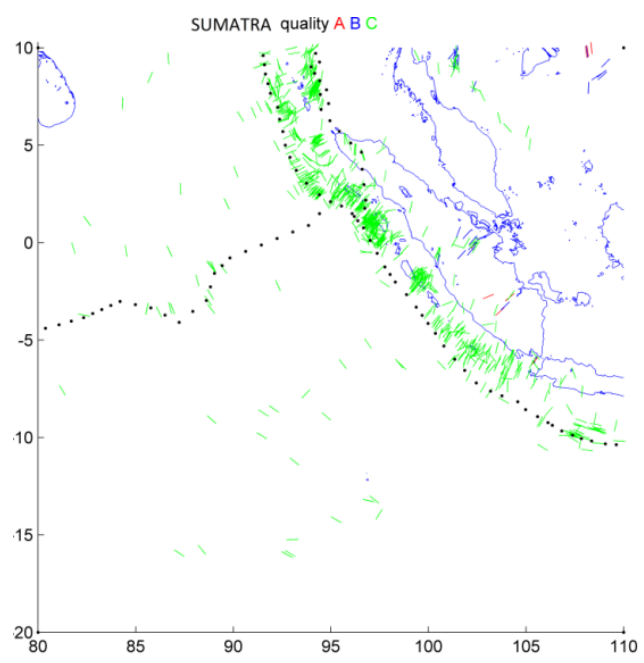

Figure 1: Sumatra region, with WSM data on stress orientations and (short segments) bird's plate boundaries data (dots).

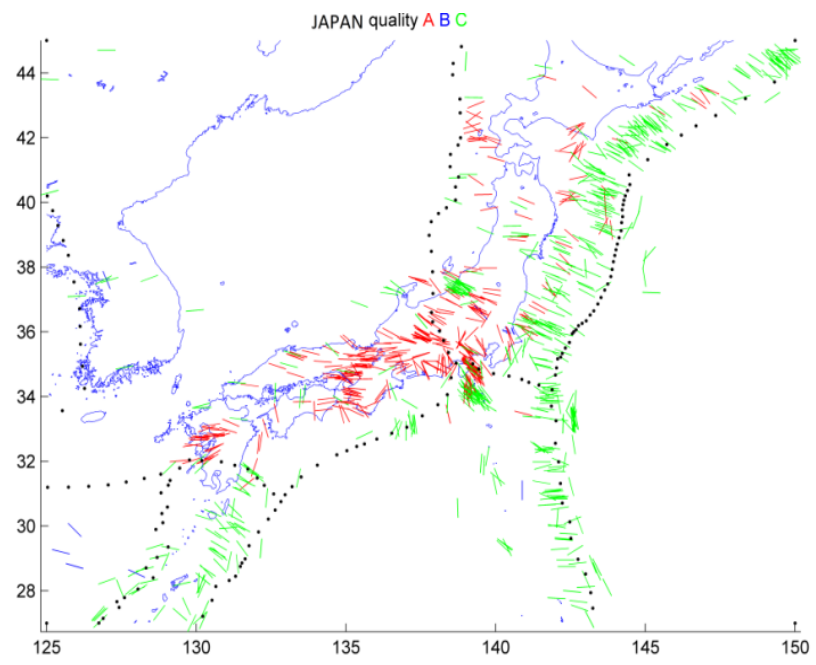

Figure 2: Japanese region with WSM data on stress orientations (short segments) and bird's plate boundaries data (dots).

- The tectonic stresses fields are analysed based on the plane stress assumption that is a consequence of the fact that one of the principal stresses is usually oriented vertically [5-7]. Therefore the 3D stress field in the Earth's crust can be decomposed into 2D plane stress and 1D, which mainly presents the effect of gravity.

- The Earth's curvature is neglected due to the sizes. 
- $\quad$ No changes in the stress fields are assumed to be before the earthquake and after it (Sumatra region) and no changes in the stress fields are assumed to be before the Japan earthquake, i.e. the change has occurred right after the earthquake.

\subsection{Mathematical model}

The 2D stress state in an elastic body in the absence of body forces can be fully described by the complex potentials $\Phi(z), \Psi(z)$ [8], where $z=x+i y$ is complex variable. The mean stress and the deviatoric stress can be determined from the complex potentials as:

$$
\begin{aligned}
& P(z, \bar{z})=2 \operatorname{Re}[\Phi(z)] \\
& D(z, \bar{z})=\bar{z} \Phi^{\prime}(z)+\Psi(z)
\end{aligned}
$$

where $\bar{z}=x-i y$ is the conjugated complex variable.

The stress deviator function can be expressed in the exponential form $D(z, \bar{z})=\left|\tau_{\max }(z, \bar{z})\right| e^{i \alpha(z, \bar{z})}$ where the modulus represents the maximum shear stress $\tau_{\max }(z, \bar{z})$ and the argument is connected with the principal directions $\theta$ as $\alpha(z, \bar{z})=-2 \theta(z, \bar{z})$ (compression is assumed to be positive). The complex conjugation stress vector on the contour is found from the stress functions as follows:

$$
\sigma_{n}(\zeta)-i \sigma_{t}(\zeta)=P(\zeta)+e^{2 i \gamma(\zeta)} D(\zeta), \zeta \in \Gamma
$$

where $\gamma$ is the angle between the tangent to the contour and the $x$-axis, $P(\zeta)$ and $D(\zeta)$ are the boundary values of the stress functions.

The stress components are found from the complex potentials by the Kolosov-Muskhelishvili formulae [8], thus the stress field is fully determined everywhere within the $2 \mathrm{D}$ body by the complex potentials.

It is assumed that the entire domain is composed from a number of subdomains. The subdomains are subdivided into triangular elements. The collocation method is used to ensure continuity across the interfaces between the adjacent elements. For this purpose several collocation points are placed on every element interface and the continuity conditions are forced at these points as explained below. Two types of element interfaces are present: boundary element interfaces BEI (presenting the plate boundaries) and domain element interfaces $D E I$ (interfaces inside the subdomains). The collocation points placed on the DEI assume continuity of the complex potentials, while the collocation points on BEI assume continuity of the stress vector. The previous approach [3] assumed continuity of two potentials $\Psi(z), \Phi^{\prime}(z)$ across the adjacent elements, the subdomain boundaries have not been taken into account. As a result only the complex-valued stress deviator function (resp. maximum shear stress and stress trajectories) has been determined. 
In the present study the continuity equations are imposed on $\Phi(z), \Psi(z), \Phi^{\prime}(z)$ (across the elements) and the stress vector (across the plate boundaries). The approximations for the complex potentials $\Psi(z), \Phi^{\prime}(z)$ within the elements are taken in the form of linear polynomials. The approximation for $\Phi(z)$ is obtained by the integration of $\Phi^{\prime}(z)$. Thus the approximating functions are sought in the form:

$$
\Phi^{\prime}(z)=a+b z, \Phi(z)=a z+\frac{1}{2} b z^{2}+e, \Psi(z)=c+d z,
$$

These functions satisfy a-priory the governing equations within the elements. The parameters $a, b, c, d$ are complex while the parameter $e$ is real. This is due to the fact that the constant in the imaginary part of $\Phi(z)$ does not affect the stresses.

The continuity of the complex potentials yields the following set of equations:

$$
\begin{aligned}
& \left.\Phi^{\prime}\left(z_{c}\right)\right|_{n_{c}}-\left.\Phi^{\prime}\left(z_{c}\right)\right|_{m_{c}}=0 \\
& \left.\Phi\left(z_{c}\right)\right|_{n_{c}}-\left.\Phi\left(z_{c}\right)\right|_{m_{c}}=0, \\
& \left.\Psi\left(z_{c}\right)\right|_{n_{c}}-\left.\Psi\left(z_{c}\right)\right|_{m_{c}}=0
\end{aligned}
$$

where $n_{c}$ and $m_{c}$ are the numbers of adjacent elements and $z_{c}$ is the position of a particular collocation point on the boundary between these elements lying in the same subdomain, $\gamma_{c}=\gamma\left(\mathrm{z}_{c}\right)$.

Continuity of the stress vector results in the following equations:

$$
\left.P\left(z_{s}, \bar{z}_{s}\right)\right|_{n_{s}}+\left.e^{2 i \gamma_{s}} D\left(z_{s}, \bar{z}_{s}\right)\right|_{n_{s}}-\left.P\left(z_{s}, \bar{z}_{s}\right)\right|_{m_{s}}-\left.e^{2 i \gamma_{s}} D\left(z_{s}, \bar{z}_{s}\right)\right|_{m_{s}}=0,
$$

where $n_{s}$ and $m_{s}$ are the numbers of adjacent elements and $z_{s}$ is the position of a particular collocation point on the boundary between subdomains, $\gamma_{s}=\gamma\left(z_{s}\right)$.

The data on stress orientations are directly implemented in the system of linear algebraic equations, based on the fact that the maximum shear stress is a real-valued function of the complex variables. Thus, the following equation is valid at every data point:

$$
\operatorname{Im}\left[D\left(z_{j}, \bar{z}_{j}\right) e^{2 i \theta\left(z_{j}\right)}\right]=0
$$

where $N$ is the number of known data $\theta\left(z_{j}\right), j=1, \ldots, N$.

Equations (4)-(6) form the system of equations for the determination of unknown coefficients in (3). Due to the homogenous right hand side of this system, at least one extra equation has to be used to provide consistency of the system. This equation expresses normalization of the complex stress deviator by unknown positive constant that has dimension of stresses. Thus, the maximum shear stress depends upon a free multiplicative parameter $A(A>0)$, which cannot be determined in the framework of this modelling. This condition yields that the sum of all $\tau_{\max }(z, \bar{z})$ placed on the known data points is equal to the number of data points: 


$$
\sum_{j=1}^{N} D\left(z_{j}, \bar{z}_{j}\right) e^{2 i \theta\left(z_{j}\right)}=N,
$$

Thus, the complete system consists of the following equations:

- For every domain element interface's collocation point three equations providing continuity of the complex potentials eq. (4).

- For every boundary element interface's collocation point one equation providing continuity of stress vector, this presents continuity of stress vector along the fault eq. (5).

- $\quad N$ equations (6) directly implementing the data on stress orientations into the system.

- One equation averaging the maximum shear stress values at the points of measurement eq. (7).

The total number of equations, $q$, is the sum of all equations above; it will be specified for particular examples considered further on. The total number of real unknowns is $9 n$, where $n$ is the number of elements.

The system of equations (4)-(7), can be written in a matrix form as $\mathbf{M r}=\mathbf{s}$. $\mathbf{M} \in R^{q \times 9 n}, \mathbf{s} \in R^{q}$ and $q>9 n$. The $9 n$ vector of unknowns $\mathbf{r}$ is formed from the unknown coefficients $a, b, c, d, e$ for each element. Right-hand side $\mathbf{s}$ is formed as $\mathbf{s} \in(0, \ldots 0, N)^{T}$. Detailed description of the matrix formation is found in Irsa and Galybin [2010]. This system is over-determined and further solved with the LSQR iterative method [9].

\section{Results of modelling}

This section presents the results of stress modelling in two regions shown in Figures 1 and 2. Apart from the stress trajectories, the other characteristics of the full stress tensor cannot be reconstructed uniquely. Thus, due to (7) the maximum shear stress is normalized such that its average over the domain is unity. The mean stress is found by integration of the differential equation of equilibrium, which introduces an additive arbitrary constant $\mathrm{B}$, therefore the stress components include two arbitrary parameters, expressed through the multiplicative and additive arbitrary constants. We compare the results of reconstructions for the Sumatra region, the CASE1 and the CASE2, by plotting the stress trajectory patterns, the normalized maximum shear stresses, the normalized mean stresses, and profiles of the normalized normal $\sigma_{n}$ and shear $\sigma_{t}$ stresses along all some interfaces.

\subsection{Sumatra region (longitude $80 \mathrm{E}-110 \mathrm{E}$, latitude $20 \mathrm{~S}-10 \mathrm{~N}$ )}

The Sumatra region contains the Sunda trench which is a subduction zone on the junction of the Sunda (SU), Burma (BU), Indian (IN) and Australian (AU) plates. Following the previous analysis [10], we analyze the region 30 by 30 degrees between $80 \mathrm{E}-110 \mathrm{E}$ and $20 \mathrm{~S}-10 \mathrm{~N}$. The plate boundaries are represented by 91 points from (PB2002). 
There are 854 data of A-C quality in total separated into 269 data for the CASE 1 and 585 data for the CASE 2. One can notice the changes of the stress orientations near the southern interface between AU-SU plates comparing these two subsets. This area includes new stress orientations data in the Sunda plate with the direction sub-parallel to the trench (perpendicular to the previously observed data). Similar changes are observed for the northern part of the BU plate inside of the selected region.

The domain is subdivided into 319 triangular elements (shown in Figure 3); among them 80 in SU, 161 in $\mathrm{AU}, 15$ in BU and 63 in IN. Every interface between the adjacent elements within a tectonic plate contains 2 collocation points. Every interface on the plate boundaries contains 3 collocation points. For the configuration shown in Figure 3, the conditions expressing continuity of the complex potentials across the adjacent elements resulted in 5042 equations of which 216 equations have been imposed by the continuity of the stress vector across the plate margins. The percentage of the data equations out of the total number of equations was $4.8 \%$ for the CASE 1 and $10 \%$ for the CASE 2 .

The data in the CASE 1 are distributed over the whole region with different density. Most of the data are located at the Sunda trench. The observed orientations are shown together with the obtained stress trajectories in Figure 4a. The reconstructed maps of maximum shear stress and mean stress are shown in Figure $4(\mathrm{~b}, \mathrm{c})$ respectively. The new data in the $C A S E 2$ are located near the plate boundaries and therefore the results of stress reconstructions in the regions remote from the plate boundaries are less accurate and have to be considered as extrapolation. This extrapolation, however, fully satisfy all the governing equations accepted in modelling. The results of the stress state reconstruction in the CASE 2 are presented in Figure 4(d-f).

Comparison of the two cases, the CASE 1 (before 26.12.2004) and the CASE 2 (after 27.12.2004), shows the change in the stress trajectory field on the AU/SU interface. One singular (isotropic) point (where the maximum shear stress vanishes) has been detected in the southern part of the Sunda Trench. The mean stress has significantly increased in this area. There is also significant decrease of the maximum shear stress after the event, particularly inside the Burma plate and alongside the interfaces of AU/SU, IN/BU and BU/SU. It should be noted that hereafter the changes in the stress field are discussed relatively to the average values over the considered region, i.e. for the functions $\tau_{\max }$ and $p$ and for the normalized boundary stresses.

The absence of new data in some areas in the CASE 2 does not affect the results of the stress reconstructions alongside the plate boundaries. The distributions of normal and shear stresses along the interfaces between all interfaces are shown in Figure 5 for both, the CASE 1 and the CASE 2. It is evident from the figures that the normal stresses have changed considerably along the AU/SU boundary after the event.

It is also evident from the distributions presented in Figure 5 that the magnitudes of the shear stresses are comparable with the normal stresses. The boundary shear stresses are often neglected in the conventional modelling, which 


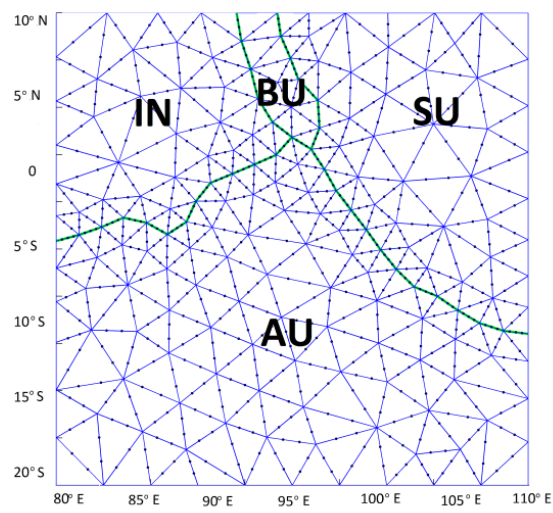

Figure 3: Sumatra region discretization. The boundary element interfaces are marked with the thick (green) line.

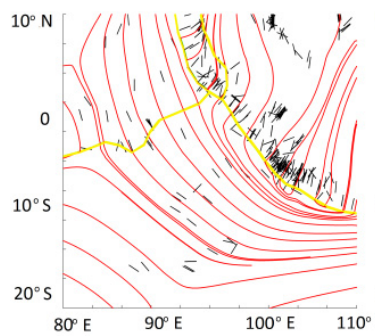

(a)

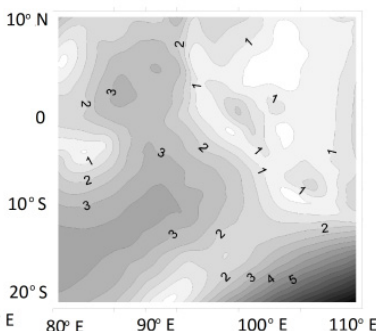

(b)

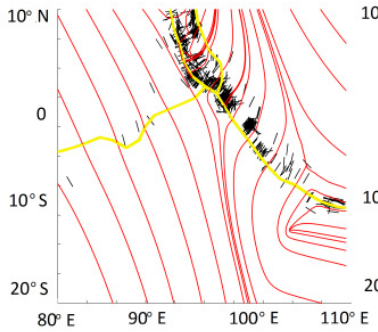

(d)

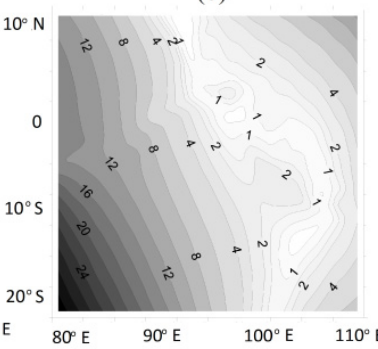

(e)

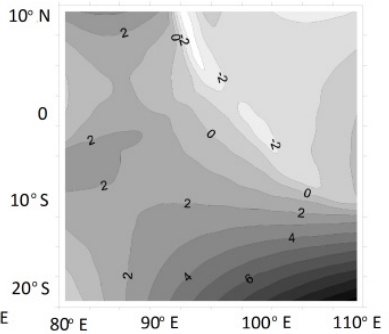

(c)

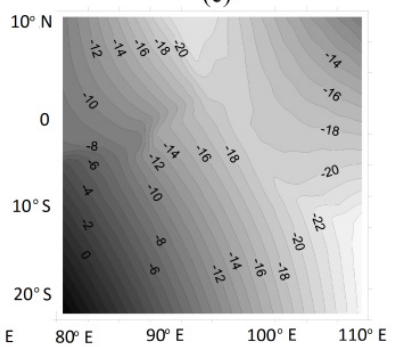

(f)

Figure 4: Stress field reconstructions in the Sunda Trench region. The CASE 1 with the data up to 26.12.2004 (a) data and stress orientations, (b) maximum shear stress, (c) mean stress; the CASE 2 with the data after 27.12.2004 (d) data and stress orientations, (e) maximum shear stress, (f) mean stress.

may result in large errors. Some parts of the boundary, e.g. AU/SU and BU/IN have been found to be mainly driven by the normal stress. 

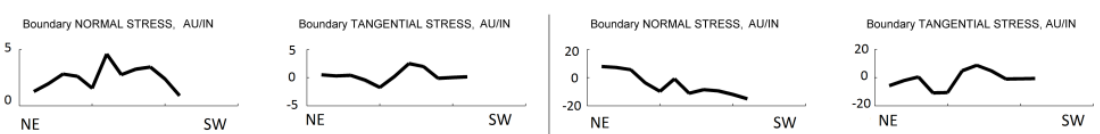

Boundary NORMAL STRESS, AU/SU
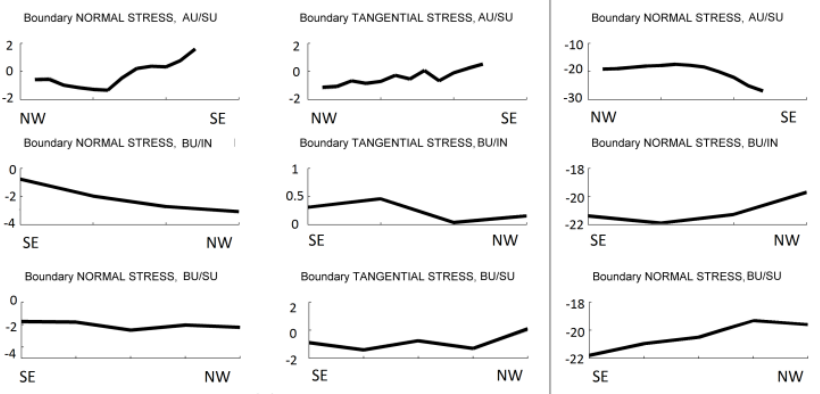

Boundary TANGENTIAL STRESS, AUISU

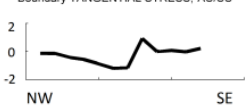

Boundary TANGENTIAL STRESS, B 0.5
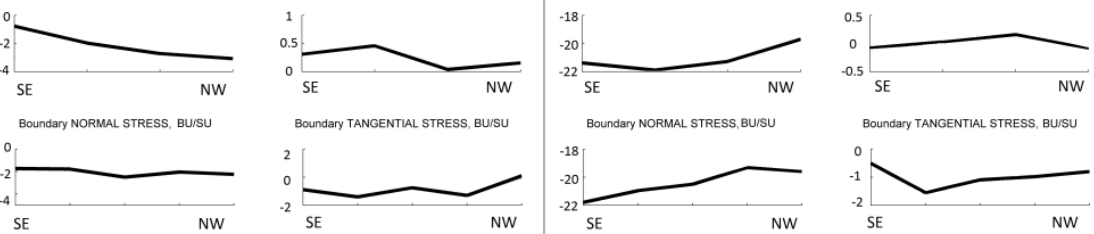

(a)

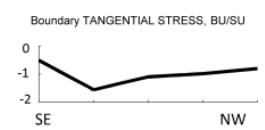

(b)

Figure 5: Reconstructed boundary stresses along the margins of the tectonic plates within the Sunda trench region: normal stress $\left(1^{\text {st }}\right.$ and $3^{\text {rd }}$ columns) and tangential stress $\left(2^{\text {nd }}\right.$ and $4^{\text {th }}$ columns), (a) CASE 1 , (b) CASE 2.

\subsection{Japanese region (longitude 125E-150E, latitude $27 \mathrm{~N}-45 \mathrm{~N}$ )}

The investigated Japanese region consists of the following tectonic plates: Pacific (PA), Okhotsk (OK), Eurasia (EU), Philippine Sea (PS), Okinawa (ON), and Yangtze (YA). The analysed region is 25 by 18 degrees between $125 \mathrm{E}-150 \mathrm{E}$ and $27 \mathrm{~N}-45 \mathrm{~N}$. The plate boundaries are represented by 165 points from (PB2002).

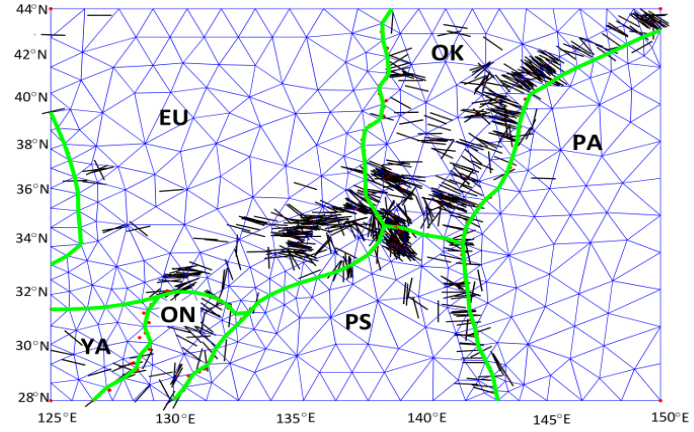

Figure 6: Japan region discretization. The boundary element interfaces are marked with the thick (green) line.

There are 830 data of A-C quality. The domain is subdivided into 722 triangular elements (shown in Figure 6). Every interface between the adjacent elements within a tectonic plate contains 2 collocation points. Every interface on the plate boundaries contains 3 collocation points. For the configuration shown 
in Figure 6, the conditions expressing continuity of the complex potentials across the adjacent elements resulted in 12839 equations of which 279 equations have been imposed by the continuity of the stress vector across the plate margins. The percentage of the data equations out of the total number of equations was $6.4 \%$.

This region contains much higher quality data compared to the previous ones (see Figures 1 and 2). The data are distributed more homogeneously and thus the results are expected to be of higher accuracy. The observed orientations are shown together with the obtained stress trajectories in Figure 7a. The reconstructed maps of maximum shear stress and mean stress are shown in Figure $7(b, c)$ respectively.

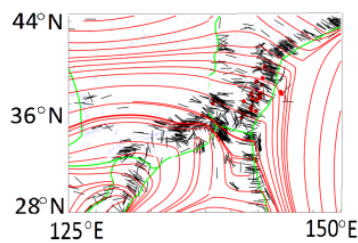

(a)

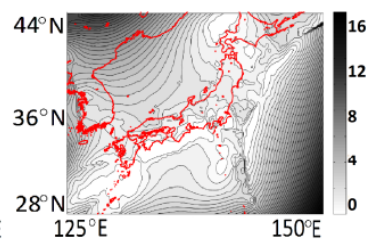

(b)

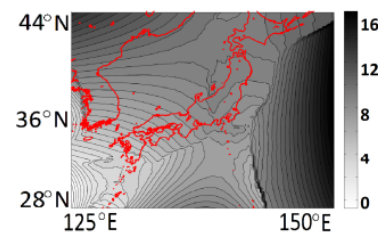

(c)

Figure 7: Stress field reconstructions in the Japanese region. (a) data and stress orientations, (b) maximum shear stress, (c) mean stress.
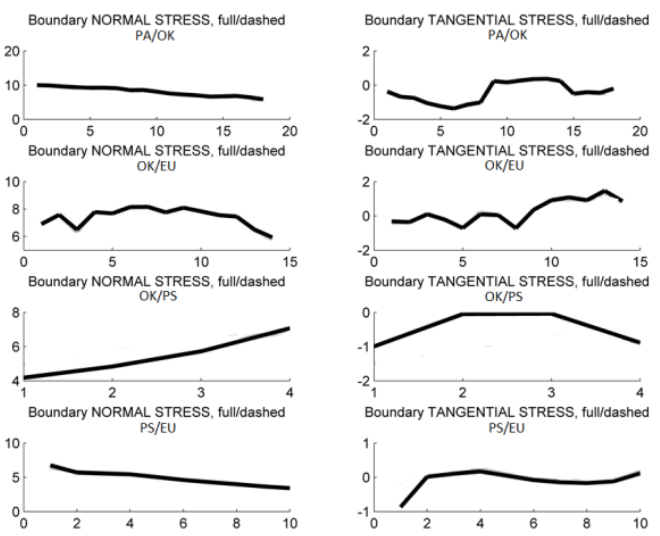

Figure 8: Reconstructed boundary stresses along the margins of the tectonic plates within the Japan region, normal stress and tangential stress.

The resulting boundary conditions are shown in Figure 8 . The boundary conditions results show that the $\mathrm{PA} / \mathrm{OK}$ boundary is mainly driven by the normal stress. This is also evident from the physical movement of the tectonic plates, which indicates correctness of the solution. It is remarkable that the analysis reveals relatively high tangential stresses for the remaining boundaries, which have similar magnitudes as the normal stresses. Therefore the commonly neglected tangential stresses are indeed important in the analyses. 
Comparison of the stress patterns of the Sumatran region before the great Sumatran earthquake and the Japan region shows very similar patterns as for stress trajectories, so does for the maximum shear stress and mean stress. The areas where the earthquakes occurred have very low, if not singular, maximum shear stress values. Also the comparison of the normal and tangential stresses on the AU/SU boundary and PA/OK boundary shows similar behaviour, i.e. the normal stresses are much greater as compared to the shear stresses. This result also reflects the directions of the physical movement of the tectonic plates.

\section{Discussion and conclusions}

Two regions, affected by similar events, have been analysed using the modification of the STEM technique developed earlier [3]. The first one was the region where the great Sumatran earthquake $(26.12 .2004,9.1 \mathrm{M})$ happened. The second one was the region of the recent Japan earthquake. Both these events are similar, not only by the magnitudes of the earthquakes and the triggered tsunamis but also by similar geodynamic conditions of tectonic plate interactions in the subduction zones.

The proposed modification of the STEM [3] takes into account both the stress orientations data from the WSM database and the tectonic plate boundaries from Birds PB2002 database. This allowed us to reconstruct the stresses inside the tectonic plates as well as the stresses along the plate margins. Overall, four outputs are obtained: the stress trajectories field (determined uniquely), the map of normalized maximum shear stress, the mean stress and boundary conditions in terms of normal and tangential stress (these fields include two arbitrary parameters).

The results of this study demonstrate that the stress fields have changed after the great Sumatran earthquake. Moreover the results show that both these regions have somewhat similar stress patterns prior to the events and, thus, the stresses in the Japan region might be expected change in a similar way as the ones in the Sumatran region after the earthquake.

\section{Acknowledgement}

The second author is grateful to RFBR for partial support of this research.

\section{References}

[1] Heidbach, O., M. Tingay, A. Barth, J. Reinecker, D. Kurfess, and B. Müller (2010), Global crustal stress pattern based on the World Stress Map database release 2008, Tectonophysics, 482 (1-4), 3-15, doi:10.1016 j.tecto.2009.07.023.

[2] Bird, P. (2003), An updated digital model of plate boundaries, Geochem. Geophys. Geosyst., 4, 1027, 55, doi:10.1029/2001GC000252. 
[3] Irsa, J., and A.N. Galybin (2010), Stress trajectories element method for stress determination from discrete data on principal directions, Eng. Anal. Bound. Elem., 34, 423-432, doi:10.1016/j.enganabound.2009.12.004.

[4] U.S. Geological Survey, obtained 20.03.2011, (http://earthquakes.usgs.gov /earthquakes/world/10_largest_world.php)

[5] Zoback, M. L., M. D. Zoback, J. Adams, M. Assumpção, S. Bell, E. A. Bergman, P. Blümling, N. R. Brereton, D. Denham, L. Ding, K. Fuchs, N. Gay, S. Gregersen, H. K. Gupta, A. Givishiani, K. Jacob, R. Klein, P. Knoll, M. Magee, J. L. Mercier, B. C. Müller, C. Paquin, K. Rajendran, O. Stephansson, G. Suarez, M. Sutter, A. Udias, Z. H. Xu, M. Zhizhin (1989), Global patterns of tectonic stress, Nature, 341, 291-298, doi:10.1038/341291a0.

[6] Amadei, B., and O. Stephansson (1997), Rock stress and its measurement, Chapman \& Hall, London.

[7] Fairhurst, C. (2003), Stress estimation in rock: a brief history and review, Int. J. Rock Mech. Min. Sci., 40, 957-973, doi:10.1016/j. ijrmms.2003.07.002.

[8] Mushkelishvili, N. I. (1953), Some basic problems of the mathematical theory of elasticity, P. Noordhoff Ltd., Groningen-Holland.

[9] Paige, Ch. C., M. A. Saunders (1982), LSQR: an algorithm for sparse linear equations and sparse least squares, ACM T. Math. Software, 8 (1), 43-71.

[10] Mukhamediev, Sh.A., and A.N. Galybin (2006), Where and how did the ruptures of December 26, 2004 and March 28, 2005 earthquakes near Sumatra originate?, Dokl. Earth Sci., 406 (1), 52-55, doi: 10.1134 /S1028334X06010132. 